\title{
Topology optimization of tungsten/copper structures for plasma-facing component applications
}

\author{
* Bailey Curzadd $[a, b]$, Alexander von Müller $[a, b]$, Rudolf Neu $[a, b]$, Udo von Toussaint $[a]$ \\ [a] Max-Planck-Institut für Plasmaphysik, Boltzmannstr. 2, 85748 Garching, Germany \\ [b] Technische Universität München, Boltzmannstr. 15, 85748 Garching, Germany \\ *bailey.curzadd@ipp.mpg.de
}

\begin{abstract}
Tungsten has established itself as the most suitable plasma-facing material for long-term operation in future magnetic-confinement fusion devices, but its properties make it a poor structural material and complicate the manufacturing of complex components. Recent advances in additivemanufacturing (AM) technology have begun to make the production of tungsten components with complex geometry more feasible. The design freedom afforded by AM could be leveraged to produce more resilient plasma-facing components (PFCs). A methodology to optimize the material distribution of composite PFCs was developed to reduce the maximum thermal stress caused by high heat fluxes. Its use was demonstrated for copper-infiltrated AM tungsten $\left(\mathrm{W}_{\mathrm{AM}} / \mathrm{Cu}\right)$ structures. Stress reductions of $50-85 \%$ are predicted under nominal load conditions. Optimized designs also reduce stress over a wide range of off-nominal conditions. The resulting optimized structures are composed of a spatially heterogeneous distribution of $\mathrm{W}$ and $\mathrm{Cu}$ comprising a broad range of composite mixtures. A sample manufacturable component was modelled based on optimization results.
\end{abstract}

\section{Highlights}

- A methodology to optimize the material distribution of composite PFCs was developed to reduce the maximum thermal stress caused by high heat fluxes.

- Stress reductions of up to $85 \%$ compared to a monolithic $\mathrm{W}$ block may be feasible with topology optimization techniques.

- Optimized component designs are effective at reducing stress even over a wide range of offnominal conditions.

- Manufacturable components can be designed based on optimization results.

\section{Keywords}

Plasma-facing component

High heat flux

Topology optimization

Tungsten/copper composite

Additive manufacturing 


\section{Introduction}

One of the major design drivers for PFCs in future magnetic-confinement fusion reactors such as ITER and DEMO is the thermal stress produced by high heat fluxes received from the plasma. The nominal steady-state heat flux expected at the ITER divertor is $10 \mathrm{MW} / \mathrm{m}^{2}$, with numerous slow transients up to $20 \mathrm{MW} / \mathrm{m}^{2}$ [1]. Additionally, plasma instabilities can cause short bursts of extreme heat fluxes which can damage the plasma-exposed surface of components [2]. Tungsten (W) is currently the most promising plasma-facing material (PFM) for armoring divertor PFCs. However, despite the many advantages of $W$ as a PFM, its brittleness makes it a poor structural material [3]. Therefore, much research has focused on combining $W$ armor with a more suitable structural material, such as copper $(\mathrm{Cu})$ alloys, as a heat sink. A divertor composed of $\mathrm{W}$ monoblocks mounted to $\mathrm{CU}$-alloy cooling tubes was developed for use in ITER. Though a comparatively simple concept such as the monoblock is believed to suffice for operation in ITER, the extreme demands placed on PFCs in future power-plant-grade devices such as DEMO have stimulated the investigation of advanced design techniques to develop more robust components [4]. One issue afflicting traditional designs is the concentration of stress at $\mathrm{W} / \mathrm{Cu}$ interfaces caused by non-uniform temperature distributions and the mismatch of the thermomechanical properties of $\mathrm{W}$ and $\mathrm{Cu}$, primarily the coefficients of thermal expansion [5]. For this reason, a variety of techniques to mitigate the stresses that develop at W/Cu interfaces in PFCs has been developed. Examples include a plain interlayer [6], a functionally graded layer [7], and a thermal break [8]. However, the effectiveness of these approaches is bound by their relatively simple geometry and limited optimization potential.

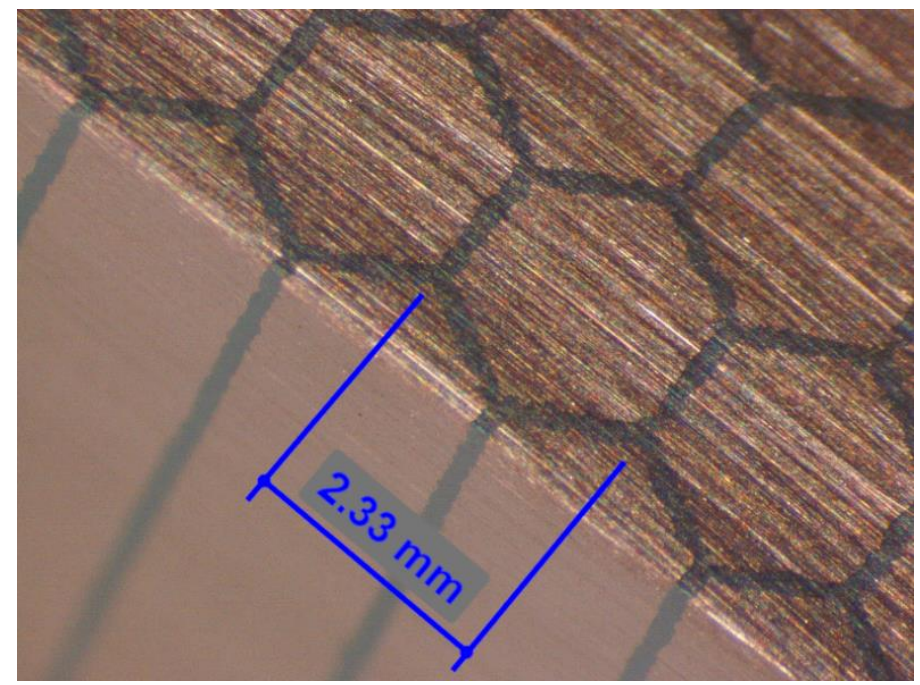

Figure 1 - Photograph of a $\mathrm{W}_{\mathrm{AM}} / \mathrm{Cu}$ composite based on an additively manufactured $\mathrm{W}$ honeycomb structure fabricated by infiltration with liquid $\mathrm{Cu}$.

Additively manufactured $W\left(W_{A M}\right)$ could be a valuable tool to overcome these limitations. AM affords substantial design freedom, which could be leveraged to design improved PFCs. Though still at an early stage of development, AM of W has been demonstrated using laser powder bed fusion (LPBF) [9]. While material with a reasonably high relative density can be consolidated, $\mathrm{W}$ is a challenging material for AM. Porosity and inter-granular cracking hamper the material's mechanical performance, which preclude its use as a monolithic structure. However, the material would be suitable as a preform employed to reinforce a ductile matrix material. W/Cu composites are currently under consideration as advanced PFC heat sink materials, and it has been demonstrated that such materials can be produced by melt-infiltration of $W_{A M}$ preforms [10]. A microsection of a $\mathrm{W}_{\mathrm{AM}} / \mathrm{Cu}$ composite based on an additively manufactured $\mathrm{W}$ honeycomb structure is illustrated in Figure 1. The present work addresses the second factor limiting the effectiveness of traditional PFC design techniques: optimization. The proposed methodology is an adaptation of structural topology 
optimization; the popularity of topology optimization has grown with the increasing use of AM and encompasses a wide range of techniques [11]. This methodology optimizes designs to reduce the peak thermal stress due to steady-state high heat fluxes by tailoring a heterogeneous $\mathrm{W} / \mathrm{Cu}$ material distribution. In the following, the proposed optimization is described, and the results of optimizing a demonstration component are presented in order to illustrate the potential of this method. Finally, the necessary steps to address current technological limitations and knowledge gaps are discussed.

\section{Optimization Approach}

\subsection{Overview}

An optimization algorithm relies on system equations to describe the behavior of the optimized physical system. The thermoelastic behavior of the optimized PFCs was described with the finiteelement (FE) method. Two FE problems must be solved to calculate stress in a thermally loaded component. First, the temperature field within the component was determined with a steady-state thermal-conduction problem. A static-equilibrium problem was solved subsequently to determine the displacements induced by thermal expansion. Knowing the temperature and displacement fields, stress was calculated. The FE formulation is detailed in Appendix A.

The PFCs were meshed with elements, and each element e was assigned a design variable $\rho_{e}$, which may vary between 0.0 and 1.0. The design variable interpolates the material composition within that element, where 0.0 corresponds to $\mathrm{Cu}$ alloy and 1.0 to pure $\mathrm{W}$. Intermediate values represent composite mixtures; the behavior of these mixtures must be determined with a model describing the elastic properties, thermal conductivity, and thermal expansion of the foreseen composite structure. These material models are described in Section 2.3. The optimization problem, detailed in Section 2.4, was formulated such that the peak von Mises stress in the component is minimized. In addition to the current stress state in the component, derivatives of stress with respect to (w.r.t.) the design variables must be calculated. This process, known as sensitivity analysis, is covered in Appendix B. Design variables of a subset of elements in the mesh can be fixed to prescribed values. This was used, for example, to guarantee a minimum thickness of pure $W$ as armor at the plasmafacing surface. Since these design variables do not change over the course of the optimization, they can be excluded from many portions of the calculation in order to reduce computation time (e.g. sensitivity analysis, optimal solution search). The remaining elements, with free design variables, comprise the design domain. Following optimization, the resulting material distribution must be realized with a manufacturable structure. Results should be considered a tool to guide the design process; the suitability of an optimized design must be subsequently verified with analysis and experimental testing.

\subsection{Demonstration component model}

Optimizations were performed using a custom-built code based on the open-source FE library libMesh [12]. The model shown in Figure 2 was chosen to demonstrate the methodology. The component was modelled in 2D with plane-stress behavior, but the optimization can be applied to 3D domains as well. The dimensions are comparable to the geometry of an ITER monoblock (see [1]), and the thermal boundary conditions are a simplified representation of typical conditions. This greatly simplified component model served to streamline the development and demonstration of the methodology; an application-specific optimization for a real component should use a model with a higher degree of fidelity to achieve the best results. Although AM and topology optimization would enable designers to re-envision even the overall form of PFCs, the use of a familiar geometry allows a more direct comparison of the new methodology with existing concepts. A $5 \mathrm{~mm}$ region facing the 
plasma was fixed to be pure $\mathrm{W}$. Estimates for the minimum thickness of armor material required to achieve an acceptable erosion lifetime vary, but values of 5 to $8 \mathrm{~mm}$ are typical [13].
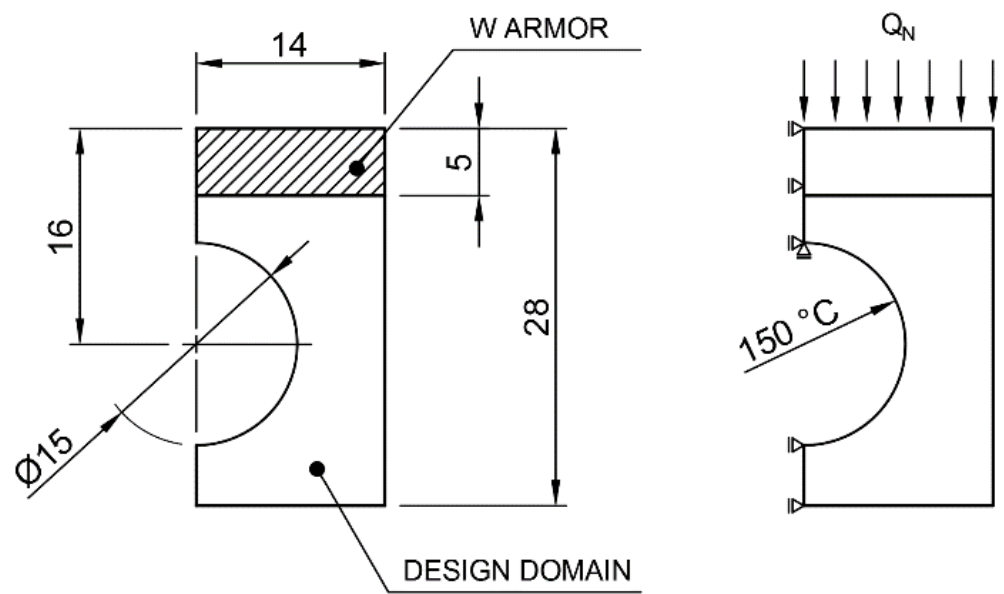

Figure 2 - Dimensions (in millimeters) and boundary conditions of the optimization domain.

The domain represents half of the component to take advantage of its symmetry, and was discretized with a procedurally generated gridded mesh; examples with $14 \times 28$ ( $1 \mathrm{~mm}$ element size) and $21 \times 42$ elements $(0.67 \mathrm{~mm}$ element size) are shown in Figure 3 . The use of a gridded mesh simplifies post-processing of results, but an unstructured mesh can be used as well, and would be necessary in some cases (e.g. to accurately represent convection boundary conditions or the geometry of a cooling tube). Elements are 4-node quadrilaterals with first-order interpolation of primary variables. A steady-state heat flux $Q_{\mathrm{N}}$ was applied at the surface of the tungsten armor, and nodes at the surface of the cooling tube were fixed to $150^{\circ} \mathrm{C}$. Calculation of thermal stresses requires knowledge of the stress state in the composite structure for at least one temperature. However, the post-manufacturing stress state of $\mathrm{W}_{\mathrm{AM}} / \mathrm{Cu}$ composites is complex, being the result of residual stress from the AM process and solidification shrinkage during melt infiltration, and has not yet been characterized. Therefore, thermal stress was calculated using a temperature $\left(\mathrm{T}_{0}\right)$ at which the material was assumed free of stress. Optimizations were performed with five stress-free reference temperatures ranging from $150^{\circ} \mathrm{C}$ to $1150^{\circ} \mathrm{C}$ in order to develop a qualitative understanding of how the optimization results depend on the residual stress state. The heat flux and stress-free reference temperature are important design parameters, and their influence on the optimized structure is discussed in detail in Section 3.
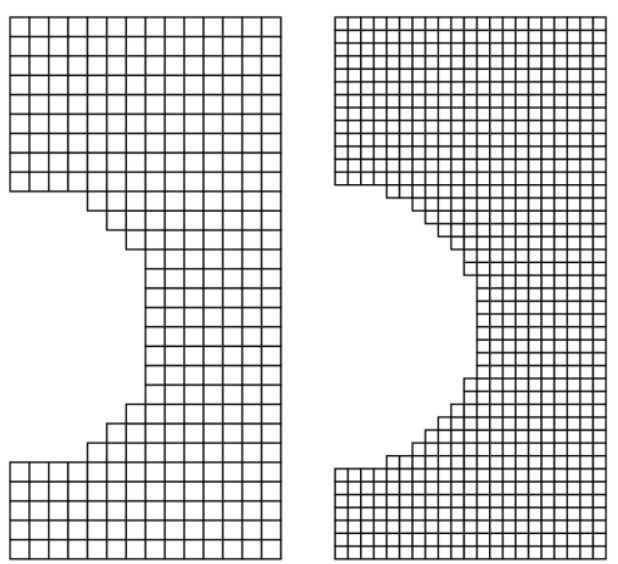

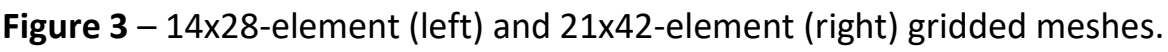




\subsection{Material models}

Due to the lack of experimental data for $\mathrm{W}_{\mathrm{AM}} / \mathrm{Cu}$ composites, material models for this work were developed using numerical homogenization. With this technique, the macroscopic properties of a composite microstructure are determined by spatial averaging of a representative volume element (unit cell) $[\mathbf{1 4 , 1 5 ]}$. Models of two composite structures were used to demonstrate and characterize the presented methodology: $\mathrm{Cu}$-alloy-infiltrated $\mathrm{W}$ regular honeycomb and $\mathrm{Cu}$-alloy-infiltrated $\mathrm{W}$ body-centered cubic (BCC) lattice. Representative volume elements are shown in Figure 4. The material models are parameterized by two quantities: elemental design variables (representing the volume fraction of $\mathrm{W}$ ) and temperature. Tabulated values of Young's modulus (E), Poisson's ratio ( $v)$, thermal conductivity $(\kappa)$, and thermal expansion $(\alpha)$ of $\mathrm{CuCrZr}$ and pure $\mathrm{W}$ at temperatures between $20^{\circ} \mathrm{C}$ and $1000^{\circ} \mathrm{C}$ were used [16]. Since the properties of $\mathrm{CuCrZr}$ are generally insufficient for the alloy to be used at high temperatures without reinforcement, tabulated values are not available up to $1000^{\circ} \mathrm{C}$. There are also locations in the pure- $\mathrm{W}$ armor region that exceed $1000^{\circ} \mathrm{C}$ at higher heat fluxes. Therefore, at temperatures above the highest tabulated values for $\mathrm{CuCrZr}$ and above $1000^{\circ} \mathrm{C}$ for $\mathrm{W}$, properties were extrapolated using a constant value. It must be stressed that this extrapolation is neither appropriate for a detailed failure analysis nor entirely suitable for application-specific optimizations. This solution was expedient for the purpose of developing and demonstrating the optimization, but should be replaced with a more thorough model of the composite and its constituents at higher temperatures, provided that this information is available. Once the operational temperature range for this class of composites has been studied experimentally, scrutiny by the designer can identify regions where the optimization yields mixtures at untenable temperatures. Properties for compositions and temperatures falling between tabulated values were found with bilinear interpolation.

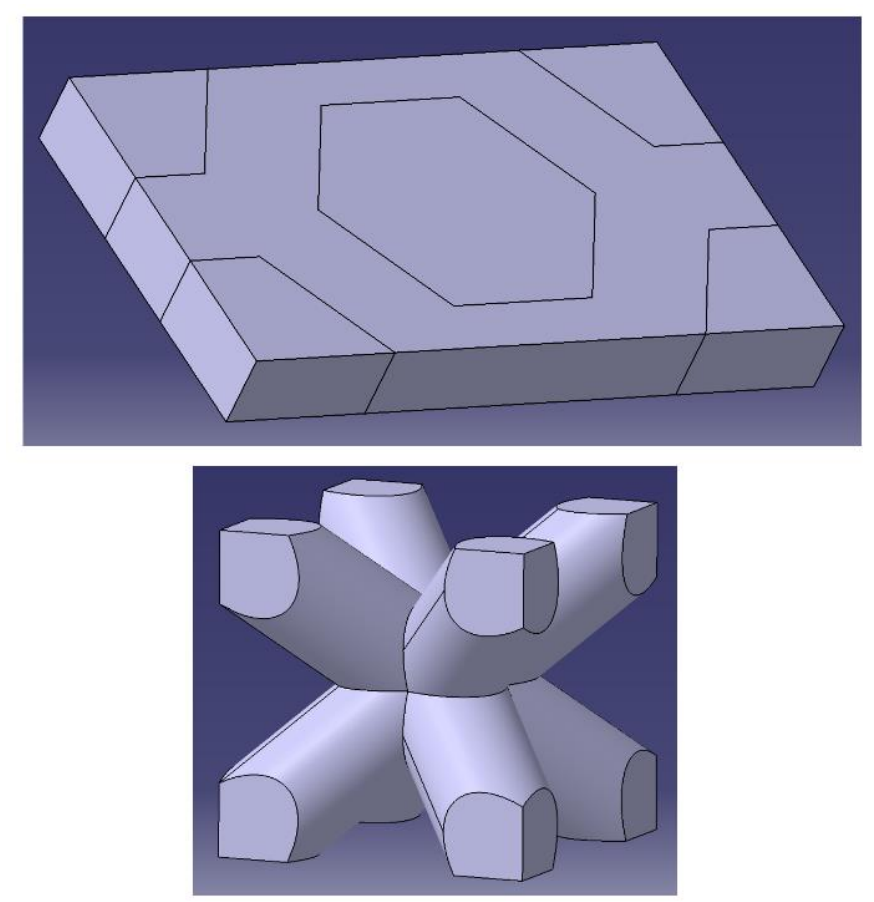

Figure 4 - Representative volume elements of regular honeycomb (above) and BCC lattice (below, Cu-alloy matrix hidden).

\subsection{The optimization problem}

Although the analysis of PFCs must consider numerous variables and failure modes, optimization techniques generally seek to minimize or maximize a single quantity. The complex stress state in a 
PFC was therefore reduced to a single quantity in two steps. First, stress at each integration point within a finite element was summarized using the von Mises equivalent stress. For a 2D, plane-stress domain, the von Mises stress was calculated with

$$
\sigma_{V}^{2}=\sigma_{1}^{2}+\sigma_{2}^{2}-\sigma_{1} \sigma_{2}+3 \tau_{12}^{2}
$$

Subsequently, the optimization problem was formulated in a way that seeks to minimize the peak von Mises stress in the domain as calculated by the macroscopic finite element models of the component. Two formulations to achieve this were developed. The first formulation aims to minimize a global stress metric $\sigma_{\mathrm{g}}$. Multiple metrics were considered. An example is the p-norm of stress values at each integration point $i$ in all $n_{\text {el }}$ elements of the mesh:

$$
\sigma_{g}=\left[\sum_{e=1}^{n_{e l}} \sum_{i=1}^{n_{i p}^{(e)}}\left(\sigma_{V}^{(e, i)}\right)^{p}\right]^{1 / p}
$$

The purpose of such metrics is to approximate the max function. For the above p-norm metric, this is done by choosing a large $\mathrm{p}$. In practice, the value of $\mathrm{p}$ is limited by the floating point representation of large powers (20 is typical). The second formulation treats stress locally. An artificial variable $\mathrm{z}$ was minimized while the mean von Mises stress in each element was individually constrained to be less than or equal to $\mathrm{z}$. Averaging of stress values within an element was performed to reduce the number of constraints in the optimization problem, as this quantity has a significant impact on computation time. This formulation is described by following min-max optimization problem:

$$
\begin{aligned}
\min & z \\
\text { subject to } & \bar{\sigma}_{V}^{(e)} \leq z, \quad e=1, \ldots, n_{e l}
\end{aligned}
$$

The Method of Moving Asymptotes (MMA) was chosen to solve the optimization problems. The MMA is a nonlinear optimization algorithm with many advantages for large, non-convex optimization problems with implicit system equations, and is popular for topology optimization $[\mathbf{1 7}, \mathbf{1 8}]$. Two forms of constraints on the material composition were tested. The first, an essential feature of traditional structural topology optimizations, constrains the global volume fraction to a maximum value:

$$
\frac{1}{n_{e l}} \sum_{e} \rho_{e} \leq \widehat{V}_{f, W}
$$

Since the proportion of elements with fixed design variables was generally substantial, this constraint was applied only to elements in the design domain in order to avoid biasing the volume fraction with elements outside the region of interest. Additionally, the standard MMA optimization problem provides individual minimum- and maximum-value constraints for each design variable:

$$
\hat{\rho}_{\min }^{(e)} \leq \rho_{e} \leq \hat{\rho}_{\max }^{(e)}
$$

The natural values for these constraints are 0.0 and 1.0, respectively. The global volume fraction constraint showed little benefit and its use is not suggested. The individual constraints have a practical use, however. The range of allowed values can be shrunken to prevent elements from developing composite structures that complicate manufacturing. For example, setting values of 0.0 
and 0.7 avoids $\mathrm{W}$-rich regions that may prevent the removal of residual powder after the LPBF process or lead to voids due to poor flow during melt-infiltration with the matrix alloy.

\section{Optimization results}

Optimizations were performed using the 14x28-element mesh in Figure 3 under a wide variety of conditions in order to illustrate the capabilities and characteristics of the presented optimization approach. The reference configuration to which optimization results were compared is a full-W 14x28-element component. Although this excludes certain features of common PFC designs, such as the copper-alloy cooling tube of the ITER monoblock design, it is useful for understanding the behavior of the algorithm. Application-specific optimizations should be compared to established real-world designs. Optimizations were performed for heat fluxes $\left(Q_{\mathrm{N}}\right)$ of 5, 10, 15, and $20 \mathrm{MW} / \mathrm{m}^{2}$. Table 1 shows the peak von Mises stress for the reference configuration at each heat flux, which was observed to be independent of the stress-free reference temperature $\left(\mathrm{T}_{0}\right)$, and Figure 5 visualizes the stress and temperature fields for a heat flux of $15 \mathrm{MW} / \mathrm{m}^{2}$. The results of optimizing with an assumed stress-free reference temperature of $150,400,650,900$, and $1150{ }^{\circ} \mathrm{C}$ were compared. The combination of 4 heat fluxes and 5 reference temperatures yielded a total of 20 load cases. Each load case is identified subsequently with a $Q_{N} / T_{0}$ designation (e.g. 10/650 represents $Q_{N}=10$ $\mathrm{MW} / \mathrm{m}^{2}$ and $\mathrm{T}_{0}=650^{\circ} \mathrm{C}$ ).

\begin{tabular}{|c|c|}
\hline$Q_{\mathrm{N}}\left[\mathrm{MW} / \mathbf{m}^{\mathbf{2}}\right]$ & $\sigma_{\max }[\mathrm{MPa}]$ \\
\hline 5 & 260.5 \\
10 & 576.1 \\
15 & 922.5 \\
20 & 1264.2 \\
\hline
\end{tabular}

Table 1 - Peak von Mises stress in a full-W domain for different heat fluxes.
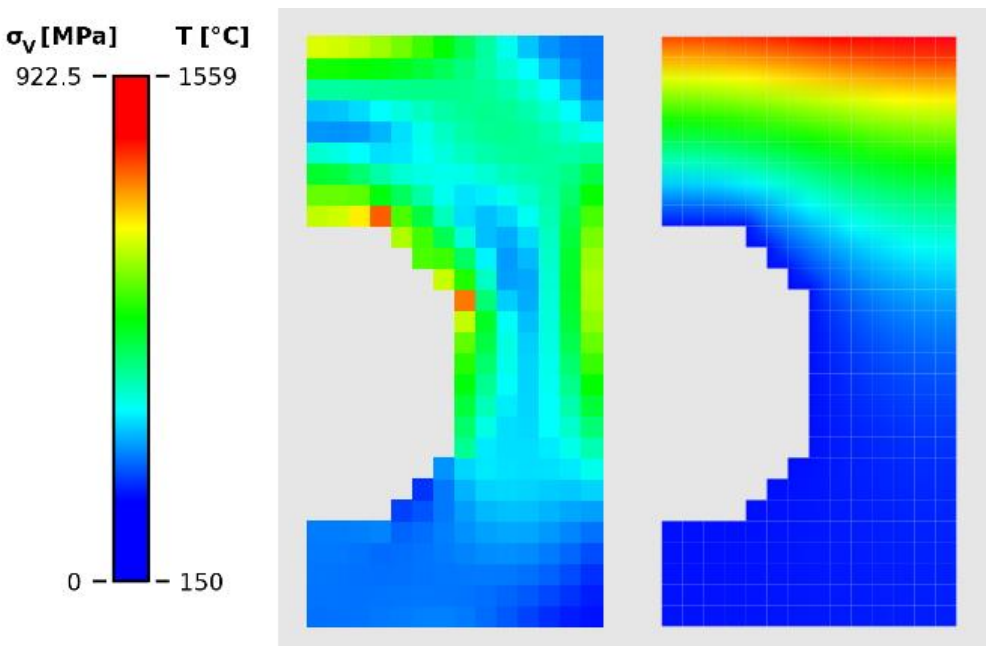

Figure 5 - Von Mises stress (left) and temperature (right) fields in the reference component with a heat flux of $15 \mathrm{MW} / \mathrm{m}^{2}$.

The optimization problem based on constrained local stress values had far superior performance to minimization of a global stress metric. While the local-stress formulation reliably achieved stress reductions and yielded manufacturable material distributions, global stress metrics converged only occasionally and often resulted in stress increases or unmanufacturable structures. All of the tested 
metrics showed similar behavior. The results presented in the following discussion were produced with the local-stress formulation.

\begin{tabular}{|cc|cc|}
\hline $\mathbf{Q}_{\mathrm{N}}\left[\mathbf{M W} / \mathbf{m}^{\mathbf{2}}\right]$ & $\left.\mathrm{T}_{0}{ }^{\circ} \mathbf{C}\right]$ & $\sigma_{\max }[\mathrm{MPa}]$ & Rel. $\Delta \sigma_{\max }$ \\
\hline 5 & 150 & 98.2 & $-62.3 \%$ \\
5 & 400 & 42.9 & $-83.5 \%$ \\
5 & 650 & 41.8 & $-84.0 \%$ \\
5 & 900 & 42.6 & $-83.6 \%$ \\
5 & 1150 & 41.6 & $-84.0 \%$ \\
\hline 10 & 150 & 219.1 & $-62.0 \%$ \\
10 & 400 & 84.9 & $-85.3 \%$ \\
10 & 650 & 82.2 & $-85.7 \%$ \\
10 & 900 & 87.2 & $-84.9 \%$ \\
10 & 1150 & 88.4 & $-84.7 \%$ \\
\hline 15 & 150 & 355.5 & $-61.5 \%$ \\
15 & 400 & 177.9 & $-80.7 \%$ \\
15 & 650 & 124.8 & $-86.5 \%$ \\
15 & 900 & 139.7 & $-84.9 \%$ \\
15 & 1150 & 139.9 & $-84.8 \%$ \\
\hline 20 & 150 & 479.2 & $-62.1 \%$ \\
20 & 400 & 370.4 & $-70.7 \%$ \\
20 & 650 & 177.1 & $-86.0 \%$ \\
20 & 900 & 185.6 & $-85.3 \%$ \\
20 & 1150 & 198.8 & $-84.3 \%$ \\
\hline
\end{tabular}

Table 2 - Peak von Mises stress and stress reduction for components optimized with a BCC lattice.

Table 2 shows the peak stress reduction achieved by the optimization for each load case using the BCC material model. A stress reduction of roughly $85 \%$ compared to the reference configuration for the corresponding heat flux was typical. Optimization with an assumed stress-free temperature of $150^{\circ} \mathrm{C}$ was less successful, but still achieved a stress reduction of roughly $62 \%$ for all heat fluxes. Figure 6 shows a progression of the material distribution and stress field for the 10/650 load case using the BCC material model. In this and all subsequent figures, blue represents $W$, and white represents $\mathrm{Cu}$ alloy. Darker shades of blue thus represent composite mixtures with higher $\mathrm{W}$ volume fractions. Within 3 iterations, the peak stress had already decreased by $76.9 \%$, and the developing structure was visible. After 10 to 15 iterations, the material distribution and stress field had largely stabilized. 


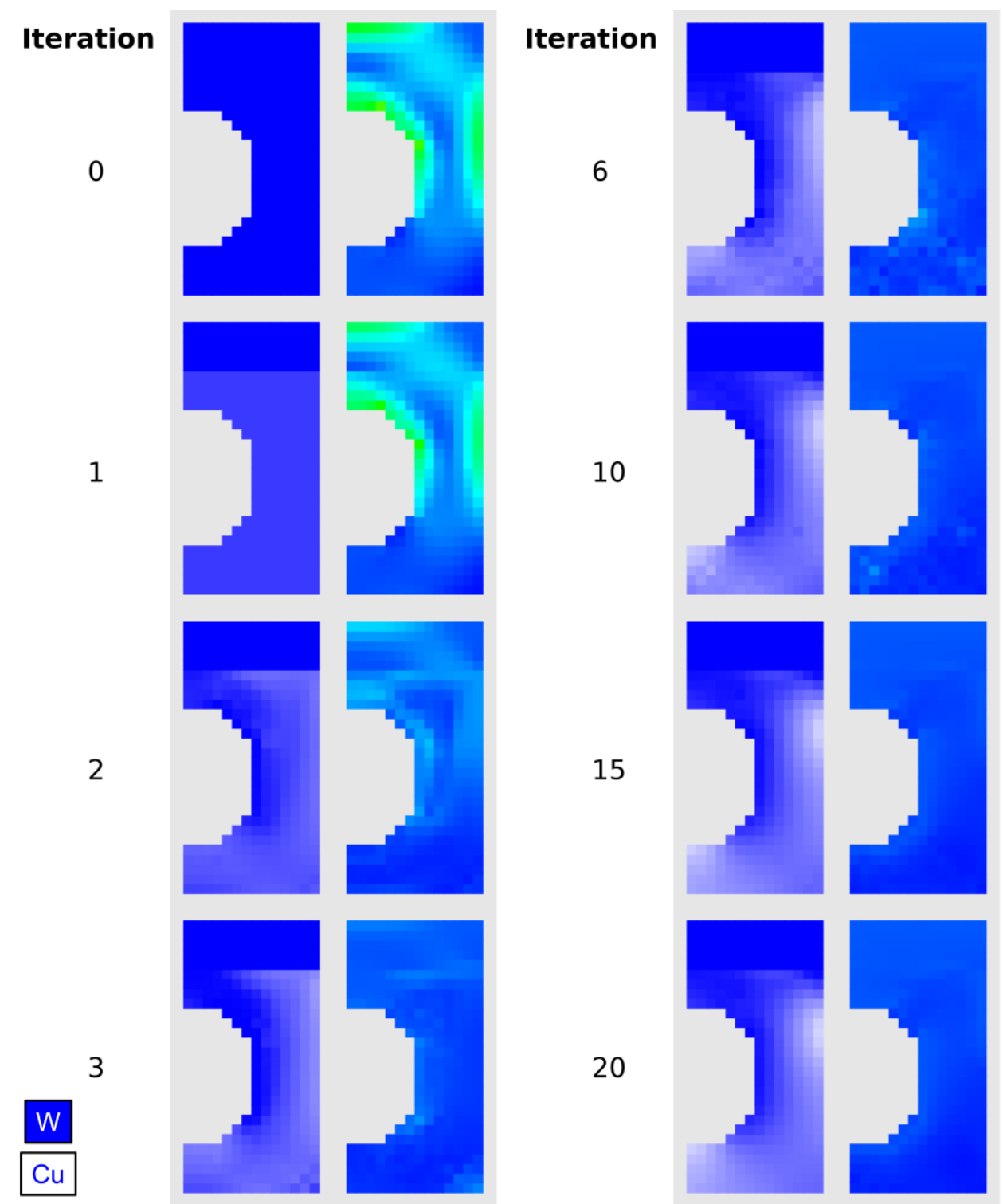

Figure 6 - Selected iterations from the optimization of a component with $\mathrm{Q}_{\mathrm{N}}=10 \mathrm{MW} / \mathrm{m}^{2}$ and $\mathrm{T}_{0}=$ $650^{\circ} \mathrm{C}$. In the material distribution (left of each pair), $\mathrm{W}$ is represented by blue and Cu alloy by white. The von Mises stress field (right of each pair) progresses to a uniform, low-stress state in fewer than 10 iterations.

Figure 7 illustrates the influence of the heat flux $Q_{N}$ on the resulting optimized structure for a reference temperature of $650^{\circ} \mathrm{C}$. Higher heat fluxes yielded designs with a greater amount of $\mathrm{Cu}$ alloy. The thermal conductivity of $\mathrm{CuCrZr}$ is significantly higher than that of $\mathrm{W}$; the optimization seemed to make use of this fact to increase the conductivity of the structure, reducing temperatures and thus thermal expansion. Qualitatively, the structures showed many similar features. A Cu-rich region developed on the side of the component, for example. This likely gave the structure a degree of compliance that allowed the hotter armor region to expand with less resistance. 


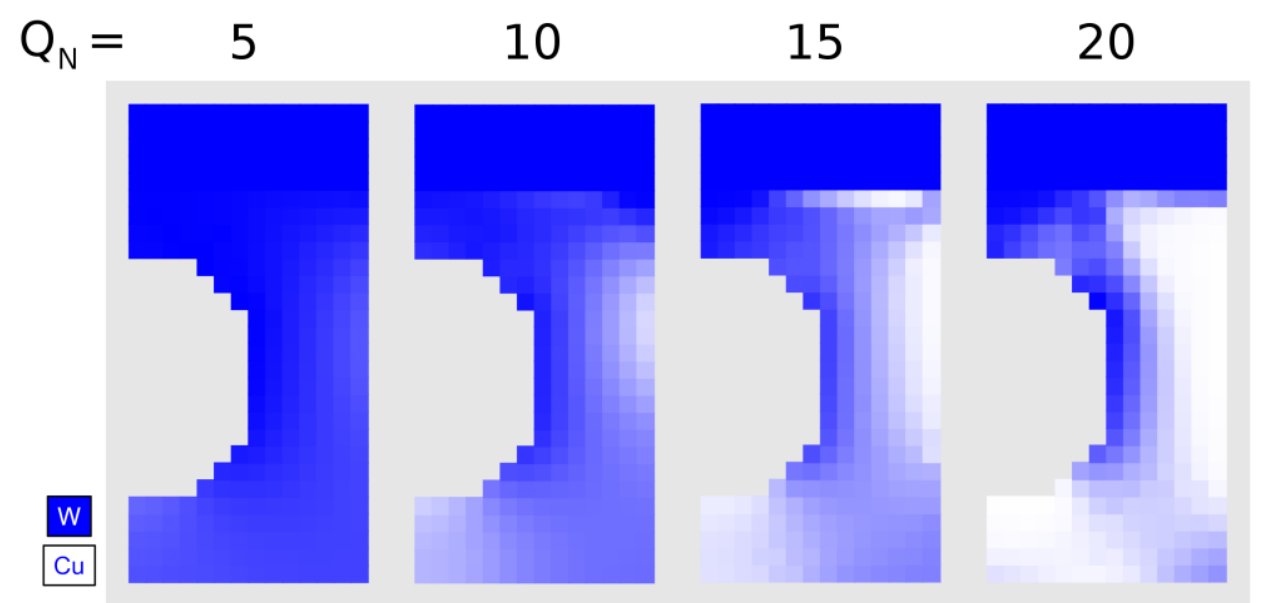

Figure 7 - Influence of the heat flux $Q_{N}$ on the resulting structure in components optimized assuming a stress-free reference temperature of $650^{\circ} \mathrm{C}$.

Figure 8 illustrates the influence of the stress-free reference temperature $\mathrm{T}_{0}$ on the resulting optimized structure for a heat flux of $10 \mathrm{MW} / \mathrm{m}^{2}$. Higher reference temperatures yielded designs with a greater amount of $\mathrm{W}$. Results for a reference temperature of $150^{\circ} \mathrm{C}$ were all qualitatively different than for the other, higher temperatures, and showed comparatively little variation for different heat fluxes. Though this correlates with the somewhat diminished stress reduction of these load cases, there is currently no intuitive explanation for this behavior.

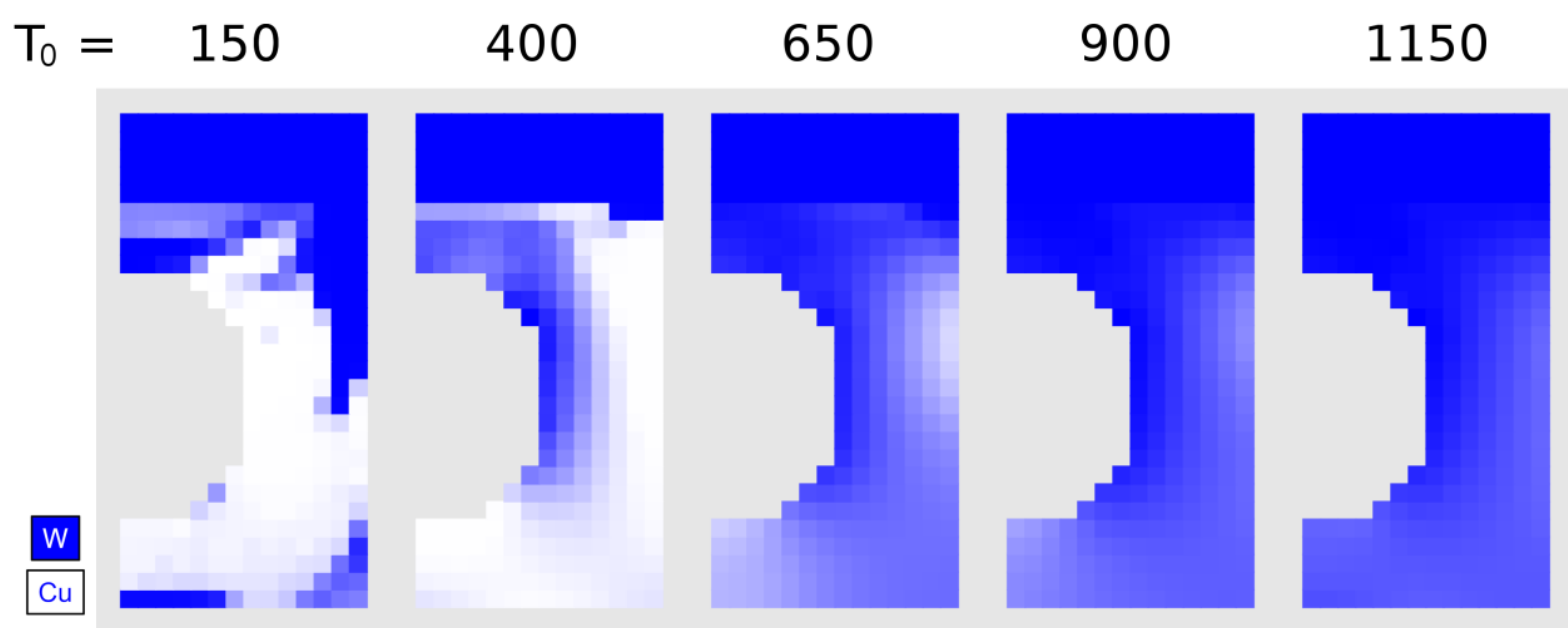

Figure 8 - Influence of the stress-free reference temperature $T_{0}$ on the resulting structure in components optimized for a heat flux of $10 \mathrm{MW} / \mathrm{m}^{2}$.

Even at a heat flux of $20 \mathrm{MW} / \mathrm{m}^{2}$, temperatures in the copper-containing regions of the design domain remained well below $1000{ }^{\circ} \mathrm{C}$. Table 3 shows the peak temperature in elements with design variables below 0.95 for optimizations with the $\mathrm{BCC}$ material model and a stress-free reference temperature of $650^{\circ} \mathrm{C}$.

\begin{tabular}{|c|cccc|}
\hline $\mathrm{Q}_{\mathrm{N}}\left[\mathrm{MW} / \mathbf{m}^{\mathbf{2}}\right]$ & 5 & 10 & 15 & 20 \\
\hline Max. $\mathrm{T}_{\mathrm{Cu}}\left[{ }^{\circ} \mathrm{C}\right]$ & 362.1 & 527.3 & 605.0 & 707.7 \\
\hline
\end{tabular}

Table 3 - The maximum temperature in copper-containing regions of designs optimized with the $\mathrm{BCC}$ material model and a stress-free reference temperature of $650^{\circ} \mathrm{C}$. 
Optimizations proved to be insensitive to the choice of material model. In addition to the BCC material model, a full set of optimizations was performed with regular honeycomb. The achievable stress reductions were comparable, and the resulting structures were similar (see Figure 9). Table 4 compares the stress reduction in a component optimized for a heat flux of $10 \mathrm{MW} / \mathrm{m}^{2}$ with the BCC and honeycomb material models.

\begin{tabular}{|c|cc|}
\hline $\left.\mathbf{T}_{0}{ }^{\circ} \mathbf{C}\right]$ & BCC & Honeycomb \\
\hline 150 & $-62.0 \%$ & $-60.7 \%$ \\
400 & $-85.3 \%$ & $-87.3 \%$ \\
650 & $-85.7 \%$ & $-86.2 \%$ \\
900 & $-84.9 \%$ & $-86.3 \%$ \\
1150 & $-84.7 \%$ & $-84.9 \%$ \\
\hline
\end{tabular}

Table 4 - Comparison of peak von Mises stress reduction in designs optimized for $10 \mathrm{MW} / \mathrm{m}^{2}$ with the BCC and honeycomb material models.

$\mathrm{BCC}$

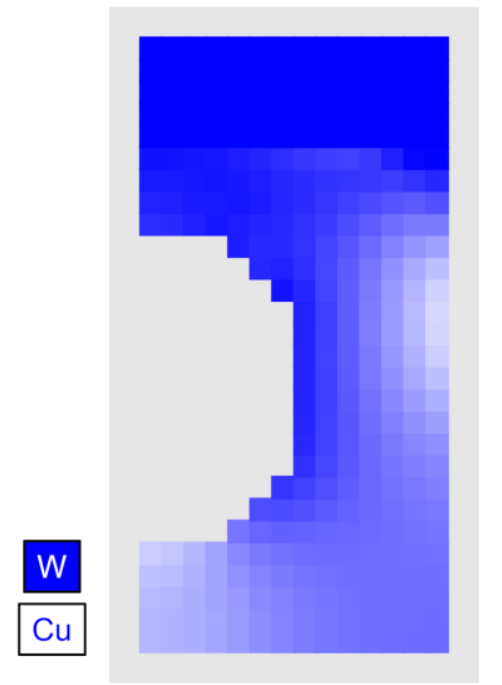

\section{Honeycomb}

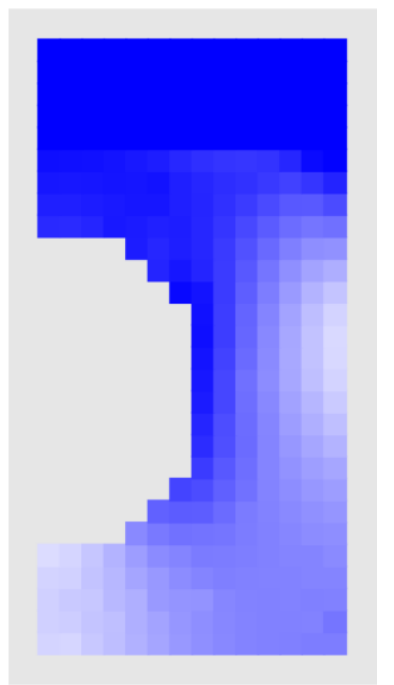

Figure 9 - Comparison of material models. Optimization results for 10/650 load case.

A component optimized for a particular load case experiences higher stresses when subjected to offnominal conditions. Table 5 compares the stress in a component optimized for the $10 / 650$ load case at each of the selected heat fluxes. Clearly, relative to nominal conditions, stress increased considerably. However, from the rightmost column, which compares the stress to the reference configuration at the corresponding heat flux, it is apparent that the optimized design remained an improvement for all heat fluxes. Even at $20 \mathrm{MW} / \mathrm{m}^{2}$, a component optimized for $10 \mathrm{MW} / \mathrm{m}^{2}$ achieved a $46 \%$ reduction of stress compared to the full-tungsten component. Notably, the component optimized for $10 \mathrm{MW} / \mathrm{m}^{2}$ performed only marginally better than the reference component at the lower heat flux of $5 \mathrm{MW} / \mathrm{m}^{2}$. Table 6 shows a similar comparison for the same optimized component assuming deviation from the assumed stress-free reference temperature. The optimized design again remained an improvement over the reference component for the considered load cases. Optimized designs only failed to surpass the reference configuration for certain load cases with large differences in both design parameters $\mathrm{Q}_{\mathrm{N}}$ and $\mathrm{T}_{0}$. Simulating the component optimized for the $10 / 650$ load case under the $5 / 1150$ conditions, for example, yielded a peak stress $182 \%$ greater than that of the full-tungsten reference at a heat flux of $5 \mathrm{MW} / \mathrm{m}^{2}$. 


\begin{tabular}{|cc|ccc|}
\hline $\mathbf{Q}_{\mathrm{N}}\left[\mathrm{MW} / \mathrm{m}^{2}\right]$ & $\mathrm{T}_{0}\left[^{\circ} \mathbf{C}\right]$ & $\sigma_{\max }[\mathrm{MPa}]$ & $\sigma_{\max } / \sigma_{\text {nom }}$ & $\sigma_{\max } / \sigma_{\text {ref }}$ \\
\hline 5 & 650 & 248.9 & $303 \%$ & $96 \%$ \\
10 & 650 & 82.2 & $100 \%$ & $14 \%$ \\
15 & 650 & 365.7 & $445 \%$ & $40 \%$ \\
20 & 650 & 679.4 & $827 \%$ & $54 \%$ \\
\hline
\end{tabular}

Table 5 - Comparison of peak von Mises stress in a BCC component optimized for the 10/650 load case at off-nominal heat fluxes $\left(Q_{N}\right)$.

\begin{tabular}{|cc|ccc|}
\hline $\mathrm{Q}_{\mathrm{N}}\left[\mathrm{MW} / \mathbf{m}^{2}\right]$ & $\mathrm{T}_{0}\left[{ }^{\circ} \mathbf{C}\right]$ & $\sigma_{\max }[\mathrm{MPa}]$ & $\sigma_{\max } / \sigma_{\text {nom }}$ & $\sigma_{\max } / \sigma_{\text {ref }}$ \\
\hline 10 & 150 & 538.1 & $655 \%$ & $93 \%$ \\
10 & 400 & 280.4 & $341 \%$ & $49 \%$ \\
10 & 650 & 82.2 & $100 \%$ & $14 \%$ \\
10 & 900 & 229.4 & $279 \%$ & $40 \%$ \\
10 & 1150 & 446.1 & $543 \%$ & $77 \%$ \\
\hline
\end{tabular}

Table 6 - Comparison of peak von Mises stress in a BCC component optimized for the 10/650 load case at off-nominal stress-free reference temperatures $\left(\mathrm{T}_{0}\right)$.

As discussed in Section 2.4, it is possible to constrain design variables on an elementwise basis, which can be used to improve the manufacturability of the optimized component. However, constraining the solution set lead to a less optimal result. Optimizations were performed with both the $\mathrm{BCC}$ and honeycomb material models while constraining design variables to the range of $0-0.5$ and $0-0.7$. Figure 10 shows the resulting structures for the 10/650 load case and Table 7 compares the peak stress to the unconstrained optimization. As expected, the peak stress increased. However, the stress reduction remained significant, at roughly $75 \%$ when constraining design variables to a maximum value of 0.7 .

\section{BCC}

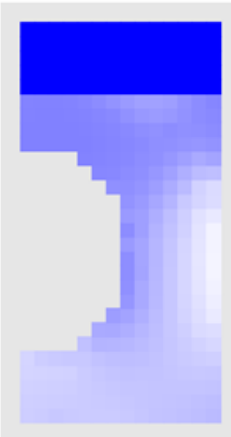

Honeycomb

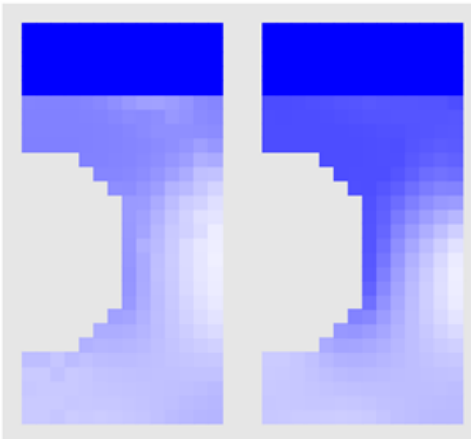

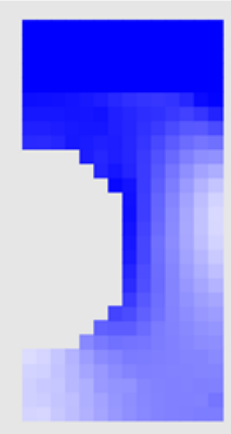

Figure 10 - Optimization results for the 10/650 load case constraining the maximum value of design variables to (from left to right for each material model) $0.5,0.7$, and 1.0.

\begin{tabular}{l|cccccc|}
\cline { 2 - 7 } & \multicolumn{1}{c}{$B C C$} & \multicolumn{5}{c|}{ Honeycomb } \\
\cline { 2 - 7 } Max. $\rho_{\mathrm{e}}$ & 0.5 & 0.7 & 1.0 & 0.5 & 0.7 & 1.0 \\
\cline { 2 - 7 }$\sigma_{\max }[\mathrm{MPa}]$ & 239.3 & 145.7 & 82.2 & 266.6 & 145.1 & 79.7 \\
\cline { 2 - 7 } Rel. $\Delta \sigma_{\max }$ & $-58.5 \%$ & $-74.7 \%$ & $-85.7 \%$ & $-53.7 \%$ & $-74.8 \%$ & $-86.2 \%$ \\
\cline { 2 - 7 } & & & & & &
\end{tabular}

Table 7 - Peak von Mises stress and relative stress reduction in components optimized for the $10 / 650$ load case with constrained design variables. 


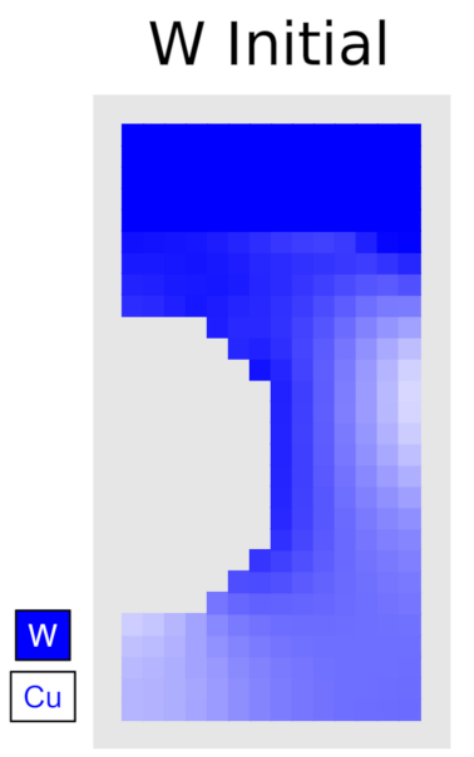

\section{Cu Initial}

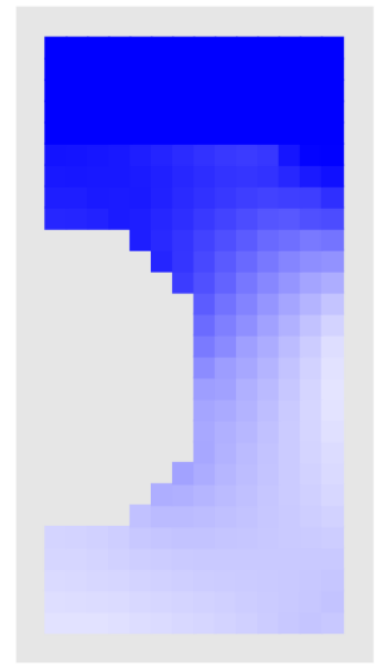

Figure 11 - Demonstration of the optimization's non-convexity with the 10/650 load case. Initialization with $1.0(\mathrm{~W}$, left) and $0.0(\mathrm{Cu}$, right) results in different structures, but both achieve a stress reduction of roughly $86 \%$.

Optimizations were performed with the BCC material model using various initial material distributions in order to investigate the optimization problem's non-convexity. Figure 11 compares the results of optimizing for the $10 / 650$ load case with the design domain initialized to 0.0 (all $\mathrm{Cu}$ ) and 1.0 (all W). The initialization biased the $\mathrm{W}$ content of the resulting material distribution. The design with all-Cu initialization had a final average volume fraction of $42.2 \% \mathrm{~W}$ in the design domain, while the all-W initialization yielded $60.5 \% \mathrm{~W}$. The peak stress was comparable, however, with both designs achieving a stress reduction of roughly $86 \%$. Mesh refinement did not fundamentally change the optimized material distribution, an issue that must be mitigated in traditional topology optimizations. Figure 12 compares the optimal design for the $10 / 400$ load case on $14 \times 28$ - and $21 \times 42$ element meshes. Though the resolution of the solutions differ, the same stress-reducing features developed regardless of element size.

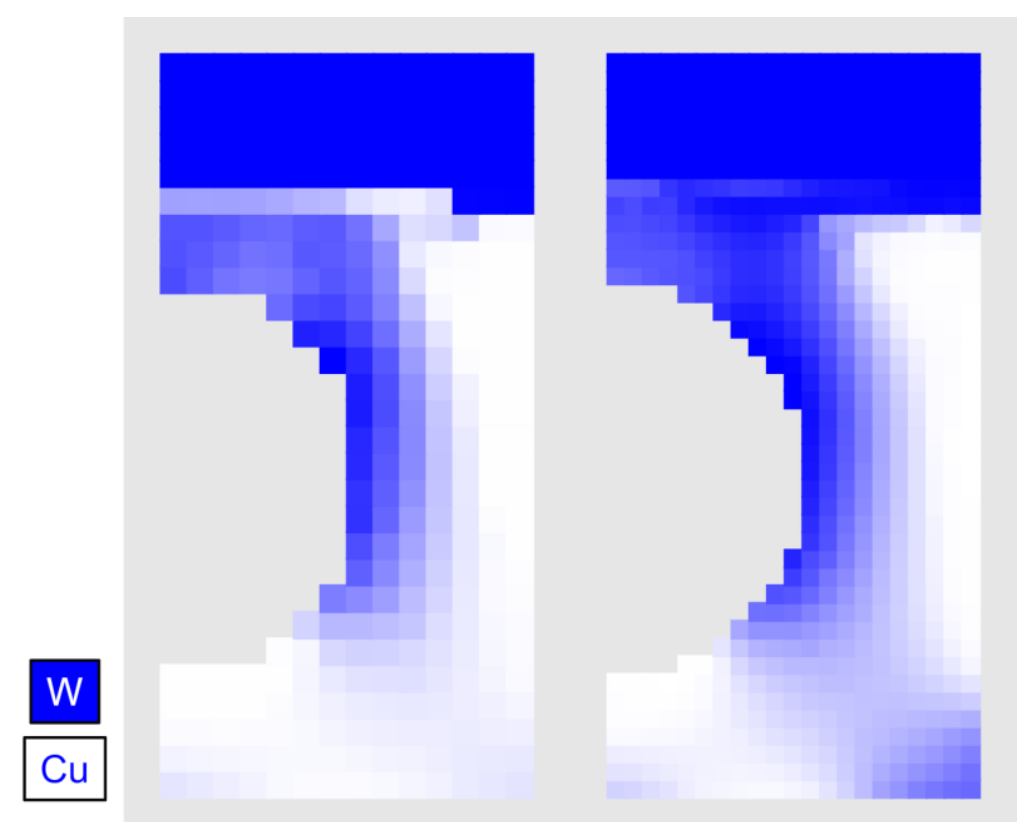

Figure 12 - Comparison of optimization results for the 10/400 load case with a $14 \times 28$ - (left) and 21x42-element (right) mesh. 
A sample topology-optimized PFC was modelled to demonstrate the feasibility of designing a manufacturable component based on optimization results. Figure 13 shows a cross-section of the component's geometry with the optimization results from which it was derived. The geometry of the BCC lattice ( $2 \mathrm{~mm}$ cell size) was generated procedurally using the scripting capabilities of the SALOME platform. Finishing touches were applied in CATIA. The volume fraction of each cell is the average of the composition in four of the square $1 \mathrm{~mm}$ elements in the $14 \times 28$-element mesh used for the optimization, and was rounded to the nearest tenth. Design variables were constrained to a maximum value of 0.7 during optimization.
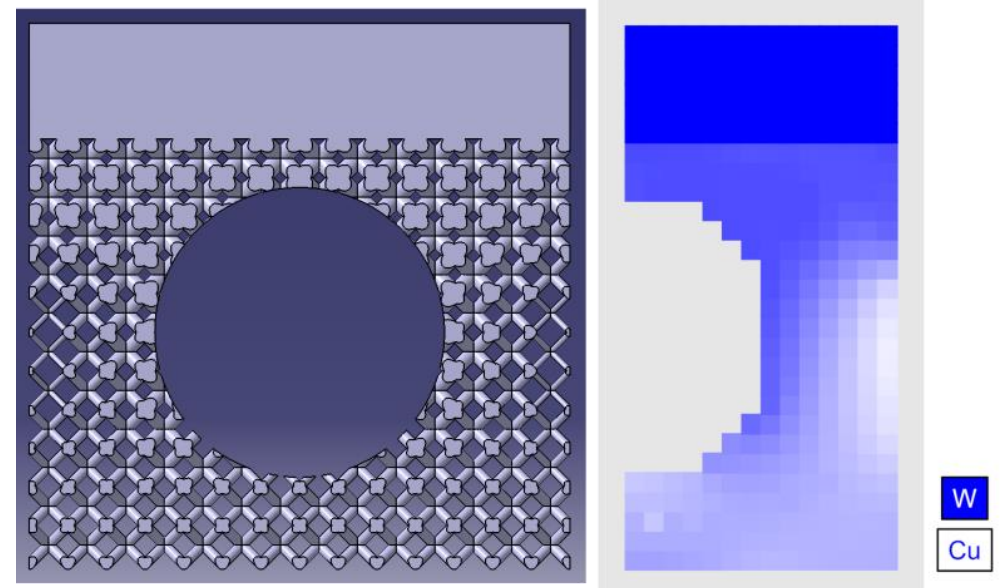

Figure 13 - A sample optimized component implemented with a BCC lattice structure (left) and the optimization results from which it was derived (right).

\section{Summary}

A design methodology for $\mathrm{W}_{\mathrm{AM}} / \mathrm{Cu}$ PFCs was developed based on structural topology optimization techniques. The optimization uses the finite-element method to describe the thermoelastic behavior of the PFC. The Method of Moving Asymptotes was used to solve a min-max optimization problem that minimizes the peak von Mises stress in the domain. The optimization currently uses temperature-dependent material models developed with numerical homogenization. Preliminary results indicate the potential for stress reductions of up to $85 \%$ compared to an all-W reference component under the conditions for which a design was optimized. Optimized designs offer improved performance for a wide range of off-nominal conditions as well.

The most significant issue currently limiting the potential of this particular optimization is a lack of experimental characterization and general understanding of $W_{A M} / C u$ composites. As seen in the results, the optimal material distribution depended strongly on the stress-free reference temperature. Thus, a prediction of the residual stress state in the composite is essential future work. Likewise, a multi-scale stress model will be necessary in order to reliably analyze failure of composite components, and mechanical testing must be performed to determine stress limits and verify properties predicted by numerical homogenization.

Despite these open issues, the promising initial results warrant both further research on this class of materials and an investigation into other, undeveloped applications of topology optimization for the design of PFCs. In this work, the peripheral geometry of optimized designs was limited to that of a traditional monoblock in order to highlight the merits of tailoring a component's material composition. This is by no means the only property of PFCs that could benefit from a reexamination with this design approach, however. Topology optimization and AM may allow designers to reenvision component shape, the form and distribution of cooling channels, etc. to achieve even further improvements. 


\section{Acknowledgements}

This work has been carried out within the framework of the EUROfusion Consortium and has received funding from the Euratom research and training programme 2014-2018 and 2019-2020 under grant agreement No 633053. The views and opinions expressed herein do not necessarily reflect those of the European Commission.

\section{Appendix A. Finite-element formulation}

The temperature field (T) in a composite PFC was modelled using the homogeneous form of Poisson's equation with variable thermal conductivity tensor $(\kappa)$ :

$$
\boldsymbol{\nabla} \cdot(\boldsymbol{\kappa} \nabla T)=0
$$

The temperature within each element was interpolated using

$$
T=\sum_{n=1}^{n_{n}} N_{n}^{(e)} \theta_{n}^{(e)}=\boldsymbol{N}^{(e)^{T}} \boldsymbol{\theta}^{(e)}
$$

where the column vectors $\mathbf{N}^{(\mathrm{e})}$ and $\boldsymbol{\theta}^{(\mathrm{e})}$ contain the element shape functions and nodal temperatures, respectively. The temperature gradient within an element was then derived from the nodal temperatures as

$$
\boldsymbol{\nabla} T=\left(\boldsymbol{\nabla} \boldsymbol{N}^{(e)^{T}}\right) \boldsymbol{\theta}^{(e)}=\boldsymbol{F}^{(e)} \boldsymbol{\theta}^{(e)}
$$

The matrix $F^{(e)}$ encapsulates the derivatives of an element's shape functions. Element conductivity matrices are built from $F^{(e)}$ and the local thermal conductivity tensor by integration over the element volume $\Omega(\mathrm{e})$ :

$$
\boldsymbol{K}_{t}^{(e)}=\int_{\Omega^{(e)}} \boldsymbol{F}^{(e)^{T}} \boldsymbol{\kappa} \boldsymbol{F}^{(e)} d \Omega
$$

Integration was performed with Gauss quadrature. Material composition was considered uniform within an element, but temperature was not. Thus, unique material properties exist at each integration point. Heat loads are applied to elements at the plasma-facing surface by integrating the prescribed heat flux $Q_{N}$ over the plasma-facing element boundary $\Gamma^{(e)}$ :

$$
\boldsymbol{f}_{q}^{(e)}=-\int_{\Gamma^{(e)}} Q_{N} \boldsymbol{N}^{(e)} d \Gamma
$$

The global conductivity matrix $\mathrm{K}_{\mathrm{t}}$ and heat load vector $\mathbf{f}_{\mathrm{q}}$ are assembled from their element-level counterparts. The temperature field was then found by solving the global system:

$$
\boldsymbol{K}_{t} \boldsymbol{\theta}=\boldsymbol{f}_{q}
$$

Due to the temperature dependence of thermal conductivity, this problem is weakly nonlinear. To account for this, the linear system was solved repeatedly until the temperature field stabilized, using updated temperatures from the most recent solution to reevaluate the thermal conductivity. This 
very simple technique converged in a negligible amount of time and generally required at most 3-4 solutions. Although no structural loads are applied directly to the simulated PFCs, stresses $(\sigma)$ develop due to non-uniform thermal expansion. These stresses are calculated using the displacements $(\mathbf{u})$ that result from the applied heat load. The system of equations for static equilibrium (without body loads) takes the form

$$
\operatorname{div} \boldsymbol{\sigma}=0
$$

With the stress tensor $\sigma$ expressed in vector form, differentiation was performed with the matrix operator D:

$$
\boldsymbol{D}^{T}=\left[\begin{array}{ccc}
\frac{\partial}{\partial x_{1}} & 0 & \frac{\partial}{\partial x_{2}} \\
0 & \frac{\partial}{\partial x_{2}} & \frac{\partial}{\partial x_{1}}
\end{array}\right]
$$

Stress in the body is proportional to the mechanical strain $\varepsilon_{\mathrm{m}}$. This strain was found by removing the contribution of thermal expansion $\boldsymbol{\varepsilon}_{\mathrm{t}}$ from the displacement-derived actual strain $\boldsymbol{\varepsilon}_{\mathrm{u}}$. Using the matrix form of the elasticity tensor $\mathbf{C}$, stress was calculated as

$$
\boldsymbol{\sigma}=\boldsymbol{C} \varepsilon_{m}=\boldsymbol{C}\left(\boldsymbol{\varepsilon}_{\mathrm{u}}-\boldsymbol{\varepsilon}_{\mathrm{t}}\right)=\boldsymbol{C}\left(\boldsymbol{D} \boldsymbol{u}-\boldsymbol{\varepsilon}_{t}\right)
$$

Using tabulated values for the mean thermal expansion from a reference temperature of $20^{\circ} \mathrm{C}$, thermal strain was expressed as

$$
\boldsymbol{\varepsilon}_{t}=\boldsymbol{\alpha}_{T}\left(T-20^{\circ} \mathrm{C}\right)-\boldsymbol{\alpha}_{T 0}\left(T_{0}-20^{\circ} \mathrm{C}\right)
$$

where $\boldsymbol{\alpha}_{\mathrm{T}}$ and $\boldsymbol{\alpha}_{\mathrm{T} 0}$ are vector representations of the (potentially anisotropic) coefficients of thermal expansion from $20^{\circ} \mathrm{C}$ to the local temperature $\mathrm{T}$ and to the stress-free reference temperature $\mathrm{T}_{0}$, respectively. Displacements are interpolated within an element using

$$
\boldsymbol{u}=\left[\begin{array}{l}
u \\
v
\end{array}\right]=\left[\begin{array}{cc}
\boldsymbol{N}^{(e)^{T}} & 0 \\
0 & \boldsymbol{N}^{(e)^{T}}
\end{array}\right] \boldsymbol{d}^{(e)}
$$

where the vector $\mathbf{d}^{(\mathrm{e})}$ contains the element's nodal displacements. After combining the differential operator D with the matrix of element shape functions to the widely used "strain-displacement matrix" B, element stiffness matrices are integrated:

$$
\boldsymbol{K}_{S}^{(e)}=\int_{\Omega^{(e)}} \boldsymbol{B}^{(e)^{T}} \boldsymbol{C} \boldsymbol{B}^{(e)} d \Omega
$$

Thermal strain was applied to an element via its load vector:

$$
\boldsymbol{f}^{(e)}=\int_{\Omega^{(e)}} \boldsymbol{B}^{(e)^{T}} \boldsymbol{C} \boldsymbol{\varepsilon}_{t} d \Omega
$$

The assembled global system was then solved:

$$
\boldsymbol{K}_{s} \boldsymbol{d}=\boldsymbol{f}
$$




\section{Appendix B. Sensitivity analysis}

Like many optimization algorithms, the MMA uses the derivatives of objective and constraint functions to determine a search direction. Evaluating derivatives for thermoelastic topology optimization is a multi-step process, and represents a significant portion of computation time in each iteration of the optimization. Due to the temperature dependence of material properties, the properties within an element not only depend on the design variable assigned to that element, but are also coupled to other design variables to some degree through the temperature field. For example, the total derivative of the elasticity matrix would be

$$
\frac{D \boldsymbol{C}}{D \rho_{j}}=\delta_{j e} \frac{\partial \boldsymbol{C}}{\partial \rho_{e}}+\frac{\partial \boldsymbol{C}}{\partial T}\left(\boldsymbol{N}^{(e)^{T}} \frac{\partial \boldsymbol{\theta}^{(e)}}{\partial \rho_{j}}\right)
$$

where $\delta_{\mathrm{je}}$ is the Kronecker delta. However, calculation of total derivatives is a very time-consuming operation. Testing showed that this process was also generally unnecessary, since material models for $\mathrm{W}_{\mathrm{AM}} / \mathrm{Cu}$ composites exhibit a much weaker dependence on temperature than on the element design variables. Approximating total derivatives of an element's material properties with partial derivatives w.r.t. its own design variable eliminated the dependence on the design variables of other elements in the mesh and yielded a dramatic decrease in computation time, no change in convergence behavior, and negligibly different optimization results. Sensitivities of the element conductivity and stiffness matrices then became

$$
\frac{\partial \boldsymbol{K}_{t}^{(e)}}{\partial \rho_{j}}=\delta_{j e} \int_{\Omega^{(e)}} \boldsymbol{F}^{(e)^{T}}\left(\frac{\partial \boldsymbol{\kappa}}{\partial \rho_{e}}\right) \boldsymbol{F}^{(e)} d \Omega
$$

and

$$
\frac{\partial \boldsymbol{K}_{s}^{(e)}}{\partial \rho_{j}}=\delta_{j e} \int_{\Omega^{(e)}} \boldsymbol{B}^{(e)^{T}}\left(\frac{\partial \boldsymbol{C}}{\partial \rho_{e}}\right) \boldsymbol{B}^{(e)} d \Omega
$$

respectively. Note that the element shape functions depend only on the mesh discretization; their derivatives do not appear in the sensitivity analysis. The sensitivity of the temperature field was calculated with

$$
\frac{\partial \boldsymbol{\theta}}{\partial \rho_{j}}=-\boldsymbol{K}_{t}^{-1}\left[\left(\frac{\partial \boldsymbol{K}_{t}}{\partial \rho_{j}}\right) \boldsymbol{\theta}\right]
$$

The thermal load vector $\mathbf{f}_{\mathrm{q}}$ does not appear because the prescribed heat loads do not depend on the design variables. The presence of the global conductivity matrix's inverse indicates that this calculation required the solution of the thermal FE problem once for each free design variable, with the square-bracketed component of the equation applied to the system as a pseudo-load vector. This and the subsequent sensitivity of the displacement field thus consumed a large amount of computation time. Since this problem amounts to the solution of a linear system with numerous right-hand side vectors, decomposition, storage, and reuse of the global system matrix was an efficient approach. Before calculating the sensitivities of the displacement field, the derivative of each element's static load vector $\mathrm{f}(\mathrm{e})$ was evaluated with

$$
\frac{\partial \boldsymbol{f}^{(e)}}{\partial \rho_{j}}=\int_{\Omega^{(e)}} \boldsymbol{B}^{(e)^{T}}\left[\boldsymbol{C}\left(\frac{\partial \boldsymbol{\varepsilon}_{\boldsymbol{t}}}{\partial \rho_{j}}\right)+\left(\frac{\partial \boldsymbol{C}}{\partial \rho_{j}}\right) \boldsymbol{\varepsilon}_{t}\right] d \Omega
$$


using the sensitivity of the thermal strain within the element:

$$
\frac{\partial \boldsymbol{\varepsilon}_{t}}{\partial \rho_{j}}=\boldsymbol{\alpha}_{T}\left(\boldsymbol{N}^{(e)^{T}} \frac{\partial \boldsymbol{\theta}^{(e)}}{\partial \rho_{j}}\right)+\delta_{j e} \frac{\partial \boldsymbol{\alpha}_{T}}{\partial \rho_{e}}\left(\boldsymbol{N}^{(e)^{T}} \boldsymbol{\theta}^{(e)}-20^{\circ} \mathrm{C}\right)-\delta_{j e} \frac{\partial \boldsymbol{\alpha}_{T_{0}}}{\partial \rho_{e}}\left(T_{0}-20^{\circ} \mathrm{C}\right)
$$

As for the temperature field, the sensitivities of the displacement field were calculated by solving the FE system with a pseudo-load vector:

$$
\frac{\partial \boldsymbol{d}}{\partial \rho_{j}}=\boldsymbol{K}_{s}^{-1}\left[\frac{\partial \boldsymbol{f}}{\partial \rho_{j}}-\left(\frac{\partial \boldsymbol{K}_{s}}{\partial \rho_{j}}\right) \boldsymbol{d}\right]
$$

Finally, the derivative of the stress tensor was determined with

$$
\frac{\partial \boldsymbol{\sigma}}{\partial \rho_{j}}=\delta_{j e} \frac{\partial \boldsymbol{C}}{\partial \rho_{j}}\left(\boldsymbol{B}^{(e)} \boldsymbol{d}^{(e)}-\boldsymbol{\varepsilon}_{t}\right)+\boldsymbol{C}\left(\boldsymbol{B}^{(e)} \frac{\partial \boldsymbol{d}^{(e)}}{\partial \rho_{j}}-\frac{\partial \boldsymbol{\varepsilon}_{t}}{\partial \rho_{j}}\right)
$$

and that of the von Mises stress followed:

$$
2 \sigma_{V}\left(\frac{\partial \sigma_{V}}{\partial \rho_{j}}\right)=2 \sigma_{1}\left(\frac{\partial \sigma_{1}}{\partial \rho_{j}}\right)+2 \sigma_{2}\left(\frac{\partial \sigma_{2}}{\partial \rho_{j}}\right)-\sigma_{1}\left(\frac{\partial \sigma_{2}}{\partial \rho_{j}}\right)-\left(\frac{\partial \sigma_{1}}{\partial \rho_{j}}\right) \sigma_{2}+6 \tau_{12}\left(\frac{\partial \tau_{12}}{\partial \rho_{j}}\right)
$$

With all of the prerequisite derivatives calculated, derivatives of the global stress metrics, e.g.

$$
\frac{\partial \sigma_{g}}{\partial \rho_{j}}=\frac{1}{p} \sigma_{g}^{1-p} \cdot\left[\sum_{e=1}^{n_{e l}} \sum_{i=1}^{n_{i p}^{(e)}} p\left(\sigma_{V}^{(e, i)}\right)^{p-1} \cdot \frac{\partial{\sigma_{V}}^{(e, i)}}{\partial \rho_{j}}\right]
$$

and average element stress values

$$
\frac{\partial \bar{\sigma}_{V}^{(e)}}{\partial \rho_{j}}=\frac{1}{n_{i p}} \sum_{i=1}^{n_{i p}} \frac{\partial \sigma_{V}}{\partial \rho_{j}}
$$

were assembled. Traditional structural topology optimizations require "filtering" of sensitivities - a process of averaging derivatives in an element with those of neighboring elements - to ensure mesh independency and to prevent unrealistic structures such as checkerboard patterns. This procedure was not only unnecessary with the presented optimization, but had no meaningful impact on results. 


\section{References}

[1] T. Hirai, et al. ITER tungsten divertor design development and qualification program. Fusion Engineering and Design 88 (2013) 1798-1801.

[2] R.A. Pitts, et al. Physics conclusions in support of ITER W divertor monoblock shaping. Nuclear Materials and Energy 12 (2017) 60-74.

[3] V. Phillips. Tungsten as material for plasma-facing components in fusion devices. Journal of Nuclear Materials 415 (2011) S2-S9.

[4] T. Barrett, et al. Progress in the engineering design and assessment of the European DEMO first wall and divertor plasma facing components. Fusion Engineering and Design 109-111 (2016) 917924.

[5] M. Li, E. Werner, and J.-H. You. Fracture mechanical analysis of tungsten armor failure of a watercooled divertor target. Fusion Engineering and Design 89 (2014) 2716-2725.

[6] A. Herrmann, et al. Design and evaluation of an optimized W/Cu interlayer for W monoblock components. Fusion Engineering and Design 86 (2011) 27-32.

[7] J.-H. You, et al. Thermal and mechanical properties of infiltrated $\mathrm{W} / \mathrm{CuCrZr}$ composite materials for functionally graded heat sink application. Journal of Nuclear Materials 438 (2013) 1-6.

[8] M. Fursdon, et al. The development and testing of the thermal break divertor monoblock target design delivering $20 \mathrm{MW} / \mathrm{m}^{2}$ heat load capability. Physica Scripta (2017).

[9] A. v. Müller, et al. Additive manufacturing of pure tungsten by means of selective laser beam melting with substrate preheating temperatures up to $1000^{\circ} \mathrm{C}$. Nuclear Materials and Energy 19 (2019) 184-188.

[10] A. v. Müller, et al. Melt infiltrated tungsten-copper composites as advanced heat sink materials for plasma facing components of future nuclear fusion devices. Fusion Engineering and Design 124 (2017) 455-459.

[11] O. Sigmund and K. Maute. Topology optimization approaches: a comparative review. Structural and Multidisciplinary Optimization 48 (2013) 1031-1055.

[12] B. S. Kirk, J. W. Peterson, R. H. Stogner, and G. F. Carey. libMesh: A C++ Library for Parallel Adaptive Mesh Refinement/Coarsening Simulations. Engineering with Computers 22 (2006) 237-254.

[13] J.-H. You, et al. European divertor target concepts for DEMO: Design rationales and high heat flux performance. Nuclear Materials and Energy 16 (2018) 1-11.

[14] B. Hassani and E. Hinton. A review of homogenization and topology optimization Ihomogenization theory for media with periodic structure. Computers and Structures 69 (1998) 707717.

[15] B. Hassani and E. Hinton. A review of homogenization and topology optimization II - analytical and numerical solution of homogenization equations. Computers and Structures 69 (1998) 719-738. 
[16] Appendix A, Materials Design Limit Data, ITER 222RLN v3.3 (2013).

[17] K. Svanberg. The method of moving asymptotes - a new method for structural optimization. International Journal for Numerical Methods in Engineering 24 (1987) 359-373.

[18] K. Svanberg. MMA and GCMMA - two methods for nonlinear optimization. https://people.kth.se/ krille/mmagcmma.pdf. 